\title{
Professionalisierung lebensweltlicher Krisen durch Technik?
}

\author{
Zur Betreuung demenziell erkrankter Personen mittels sozial \\ assistiver Robotik
}

\author{
Michaela Pfadenhauer $\cdot$ Christoph Dukat
}

(C) Der/die Autor(en) 2016. Dieser Artikel ist eine Open-Access-Publikation.

Zusammenfassung Vor dem Hintergrund unseres Interesses an sozio-technischen Pflege-Arrangements untersuchen wir, wie ,,sozial assistive“ Roboter in der stationären Altenpflege bzw. -betreuung und hier insbesondere bei demenziell erkrankten Personen eingesetzt wird. Die empirische Basis bildet eine ethnographische Langzeitforschung in einem Altenpflegezentrum, in dem aktuell zwei Exemplare des von uns untersuchten Roboters zum Einsatz kommen. Eingesetzt werden sie hier ausschließlich von „,zusätzlichen Betreuungskräften“ nach § 87b Abs. 3 SGB XI. In der Annahme, dass es die gesellschaftliche Konstruktion einer Krise ist, die einen Professionalisierungsprozess ins Rollen bringt, beschäftigt uns die Frage, welche Bedeutung Technik - nicht im Verstande von Handlungs- oder Kulturtechnik, sondern von Sachtechnik - im Rahmen einer solchen Entwicklung zukommen könnte. Der Einsatz von Robotern bei der Betreuung demenziell erkrankter Personen und ihre (instrumentelle) Handhabung durch das Betreuungspersonal lassen sowohl spezifische Formen der Krisenkonstruktion als auch besondere Formen kompetenter Krisenbewältigungsstrategien erkennen. Beide Aspekte zeigen Professionalisierungspotentiale an.

Schlüsselwörter Krise $\cdot$ Demenz $\cdot$ Pflege $\cdot$ Soziale Robotik $\cdot$ Technik . Professionalisierung · Wissenssoziologie

Dieser Artikel beruht auf dem gleichnamigen Vortrag in der Sektion Professionssoziologie beim 37. Kongress der Deutschen Gesellschaft für Soziologie 2014 in Trier.

M. Pfadenhauer $(\bowtie)$

Institut für Soziologie, Universität Wien, Rooseveltplatz 2, 1090 Wien, Österreich

E-Mail: michaela.pfadenhauer@univie.ac.at

C. Dukat $(\bowtie)$

Institut für Soziologie, Medien- und Kulturwissenschaften, Karlsruher Institut für Technologie (KIT), Kollegium am Schloss Geb. 20.12, Schlossbezirk 12, 76131 Karlsruhe, Deutschland

E-Mail: christoph.dukat@kit.edu 


\title{
Professionalization of lifeworld crises by means of technology?
}

On the use of socially assistive robots in dementia care

\begin{abstract}
Within the context of our wider interest in the socio-technical aspects of care, in this article we examine how socially assistive robots are being used in inpatient geriatric care of persons suffering from dementia. Based on an ethnographic long-term study conducted in a nursing home which currently employs two of the robots investigated by us, we focus on the behavior of those who operate these robots: so-called "additional nurses" ( $\$ 87$ b Abs. 3 SGB XI). Proceeding from the assumption that processes of professionalization are frequently triggered by (what societies define as) crises, we inquire what role technology plays in this regard. Thinking about the use of robots in dementia care, we argue, helps us understand how crises are being constructed as well as overcome - which indicates a significant potential of professionalization on the part of the "additional nurses."
\end{abstract}

Keywords Crisis - Dementia $\cdot$ Care $\cdot$ Social robotics $\cdot$ Technology ·

Professionalization $\cdot$ Sociology of knowledge

Man kann die Geschichte der modernen Gesellschaft unter anderem auch schreiben als Geschichte der Ablösung von Laienlösungen durch Formen rationalisierter Expertenlösungen von Problemen.

(Luckmann und Sprondel 1972, S. 15)

\section{Einleitung}

Es ist ein Signum moderner Gesellschaften, dass für immer mehr Handlungsund Lebensprobleme, die ehemals im familialen, verwandtschaftlichen und nachbarschaftlichen Verbund bewältigt wurden, Expertenlösungen nicht nur angeboten, sondern auch nachgefragt werden: „Der Gang zum Erziehungs- und Eheberater oder dem Psychiater wird zunehmend für viele fast so selbstverständlich wie die Inanspruchnahme des ärztlichen Fachwissens im Krankheitsfall“" (Luckmann und Sprondel 1972, S. 16). Die Bewältigung von immer mehr lebenspraktischen Krisen wird in der Moderne also an Personen abgegeben, die dies ,,von Berufs wegen“ tun, d. h. dafür ausgebildet sind und damit ihrerseits ihre materielle Existenz sichern.

Dem geht historisch ein langer Prozess der Arbeitsteilung voraus, in deren $\mathrm{Zu}$ ge sich Allgemein- und Sonderwissensbestände auseinanderentwickeln und Letztere sich fortlaufend ausdifferenzieren, womit Spezialisierungen möglich und notwendig zugleich werden (vgl. Schütz und Luckmann 2003 [1975], Kap. IV C; Pfadenhauer 2003, 14-30). Zusätzlich hat die Arbeitsrationalisierung als Erfolgsrezept der Industrialisierung Einzug in Lebens- und Handlungsbereiche jenseits industrieller Produktion gehalten. So hat sich z. B. für Beziehungs- und Familienprobleme ein zwar umfangreiches Leistungsspektrum etabliert, das aber in einzelne Dienstleistungsangebote wie Beratung, psychologische Betreuung, Therapie, Mediation zergliedert ist (vgl. zu Letzterem Maiwald 2004). 
In dieser Expertisierungs- bzw. Professionalisierungslogik kann auch die Demenzbetreuung gesehen werden, die 2008 in Deutschland als Qualifizierungsmaßnahme eingeführt worden ist. Angesichts dessen, dass die private bzw. familiäre Überforderung durch die Betreuung eines Angehörigen, der an Demenz ,erkrankt"1 ist, ein medialer Dauerbrenner ist, kann man dafür einen Bedarf unterstellen (vgl. Pfadenhauer 2015). Und der Nachfrage ist insofern der Boden bereitet, als die Überführung eines Angehörigen in eine professionelle Betreuung keineswegs mehr als moralische Verletzung des Generationenvertrags gilt, sondern im Gegenteil als verantwortungsvoller Umgang mit einer weder individuell noch laienhaft angemessen zu bewältigenden Krisensituation.

\section{Professionalisierung und Krise}

Alltagssprachlich befindet sich jemand in einer Krise, wenn er oder sie aus einem Problem nicht ohne Weiteres herausfindet. In diesem Verstande ist die Krise eine Übergangsphase vom Problem zur Lösung, wobei es unerheblich ist, ob der Ausweg aus der Krise selbst gefunden oder von anderen gewiesen wird. Mit einem solchen weiten Krisenverständnis ist allerdings jedes Problem eine Krise und jede Problemlösung bereits eine Krisenbewältigung.

Die gegenwärtige sowohl in der tagespolitischen Berichterstattung als auch in der Soziologie $^{2}$ grassierende Krisenmetaphorik geht einerseits mit der „Logik“ der Massenmedien und andererseits damit einher, dass Krisenhaftigkeit in der Moderne eine grundlegende Erfahrung ist (vgl. Prisching 1986; Nassehi 2012). Reinhard Koselleck (1976) bestimmt „Krisen“ als kürzer oder länger dauernde, entscheidungsoffene biographische Phasen, Martin Endreß (2014) versteht darunter sowohl pessimistisch als auch optimistisch konnotierte „Übergangssituationen“ aus irritierten Routinen heraus (vgl. dazu Hitzler 2014). Von daher liegt es nahe, „Routine“ als Komplementärbegriff zu Krise zu verstehen, wie dies der Titel „Krise der Routinen - Routinen der Krise“ des 37. Kongresses der Deutschen Gesellschaft für Soziologie nahelegt.

Genau betrachtet ist es aber nicht das Handeln, sondern das darin integrierte Wissen, das in die „Krise“ kommt. Auch im Alltag konnotiert „professionelles Handeln“ (etwas professionell gemacht zu haben) nicht einfach Routine, sondern einen angemessenen Umgang mit einer als krisenhaft angesehenen Situation. Aus dieser Sicht bildet nicht „Routine“, sondern „Kompetenz“ den Komplementärbegriff zu Krise. Der Kompetenzbegriff akzentuiert nicht nur die Angemessenheit von Handeln, sondern die Einheit von Handeln und Wissen im Verstande der neueren, integrativen Wissenssoziologie (vgl. Knoblauch 2010, 2014; Pfadenhauer 2010, 2014a). Aus dieser phänomenologisch fundierten Perspektive gründet Wissen in Erfahrung. Aber „der Sinn, aus dem das Wissen zehrt, erhält seine Bestätigung nicht aus einer ihm

\footnotetext{
1 Die Einordnung von Demenz als Krankheit ist umstritten (vgl. Whitehouse und George 2009 sowie das Resümee dieses Beitrags).

2 Jenseits dessen, dass sich die Soziologie generell als „Krisenwissenschaft“ versteht, trugen sowohl der Kongress der Österreichischen Gesellschaft für Soziologie 2013 als auch jener der Deutschen Gesellschaft für Soziologie 2014 die Krise im Titel.
} 
zugrundeliegenden Erfahrung, sondern erweist sich als relevant, weil er Handlungen leitet" (Knoblauch 2014, S. 351).

„Krisen“ gehen mit Transzendenz, d. h. damit einher, dass bisherige Erfahrungen überschritten werden. Das Ausmaß von Transzendenz bemisst sich danach, wie das in der gegenwärtigen Erfahrung angezeigte Nicht-Erfahrene erfahrbar ist: analog zum bisher Erfahrenen, nur über Anzeigen oder überhaupt nicht in persona (vgl. hierzu und zum folgenden Schütz und Luckmann 2003 [1975], S. 589 ff). Mittlere Transzendenzen sind dadurch gekennzeichnet, dass sie im Unterschied zu kleinen Transzendenzen nicht überschritten, im Unterschied zu großen Transzendenzen aber zumindest überblickt werden können, weil ,das in der Selbsterfahrung begründete ,Wissen“ (...) auf Andere übertragen wird“" (ebd., S. 607).

Phänomenologisch, d. h. vom subjektiven Erleben her betrachtet, bedrohen „Krisen“ die Alltagswirklichkeit (vgl. Schütz 2004). Die Schwere von Krisen bemisst sich an der Nähe oder Ferne zum Alltag, d.h. darin, ob die Person, für die dessen Wirklichkeitsakzent infrage gestellt ist, der natürlichen Einstellung verhaftet und damit handlungsfähig bleibt oder ob sie Alltagsrelevanzen aussetzt und sich der theoretischen Einstellung annähert. Diesem engeren Verständnis nach ist keineswegs jedes Handlungsproblem, sondern nur ein solches Problem eine Krise, bei dem Vertrautheitswissen und Gewissheiten nachhaltig erschüttert werden.

Dies schließt ein konstruktivistisches Verständnis von Krise nicht aus. Die Definition bestimmter körperlicher Manifestationen und Verhaltensweisen als Demenz und deren Qualifizierung als Krankheit ist eine gesellschaftliche Konstruktion. Davon bleibt die Konstitution einer Krise in der Erfahrung nicht unbenommen. Denn auch wenn Konstitutionsprozesse theoretisch subjektive Vorgänge sind, während Konstruktionsprozesse gesellschaftlicher Natur sind, sind sie empirisch über Internalisierung, d. h. dadurch verknüpft, dass Menschen in ein ,sozio-historisches Apriori“ gestellt sind und Sozialisationsprozesse durchlaufen (vgl. Berger und Luckmann 1969).

Die These, dass Krisen erst oder auch definiert, d. h. gesellschaftlich konstruiert werden müssen, bevor sie als solche erfahren werden, bildet den Kern der Bestimmung professionellen Handelns als Definition eines Problems zu einer Lösung, für die der definitionsmächtige Akteur einen Lösungsvorschlag hat (vgl. Pfadenhauer 2005). Genauer meint Professionalisierung die Institutionalisierung einer Problemsicht qua theoretisch-wissenschaftlicher Legitimationen, zu der in einem Tätigkeitsfeld Bewältigungsstrategien zuhanden sind, für die alleinige Zuständigkeit beansprucht wird und zu deren Ausführung nur jene berechtigt sind, die den Erwerb spezifischer Wissensbestände nachweisen können (vgl. Pfadenhauer 2003, S. 57-77).

Allerdings besteht Luhmann und Schorr (1979) zufolge die Eigentümlichkeit der Probleme, derer sich professionell angenommen werden muss, in ihrer Nicht-Technisierbarkeit, d.h. darin, dass sich zu ihrer Bearbeitung keine Routinen ausbilden lassen. Deshalb lassen sie sich der von Ulrich Oevermann (1996) revidierten Professionalisierungstheorie zufolge, die ihren Ausgang dezidiert an der Krise nimmt, auch nicht lösen, sondern nur stellvertretend deuten. Anders als in unserem Ansatz professionellen Handelns liegt hier eine Substanzannahme zugrunde: Unterstellt wird eine „objektive, tendenziell Krisen auslösende soziale Realität von materiellen Lebens- 
bedingungen, aber auch von nicht-intendierten Handlungsfolgen, von sprachlichen Erzeugungsregeln und von durch unbewusste Motivierungen welcher Art auch immer generierten Konstellationen“ (Oevermann 2006, S. 81; vgl. grundlegend dazu auch Prisching 1983).

Während es aus einer solchen Sicht möglicherweise Sinn macht, davon zu sprechen, dass es die lebensweltlichen Krisen (selber) sind, die professionalisiert werden, hebt der Professionalisierungsbegriff wissenssoziologisch-sozialkonstruktivistisch auf Rollen ab, die durch die Systematisierung eines Wissensgebietes, durch die Länge und Komplexität der (institutionell spezialisierten) Ausbildung, durch die Beglaubigung beruflicher Kompetenzen in institutionellen Kategorien und durch ein Geflecht von auf Sonderwissen bezogenen Selbst- und Fremdtypisierungen sozial verfestigt werden (vgl. Pfadenhauer 2003, S. 26; Pfadenhauer und Kunz 2010, S. 236).

In der Annahme, dass es die gesellschaftliche Konstruktion einer Krise ist, die einen solchen Professionalisierungsprozess ins Rollen bringt, beschäftigt uns im Folgenden, welche Bedeutung Technik - nicht im Verstande von Handlungs- oder Kulturtechnik, sondern von Sachtechnik, - im Rahmen einer solchen Entwicklung zukommen könnte. Der Einsatz von Robotern bei der Betreuung demenziell erkrankter Personen und ihre (instrumentelle) Handhabung durch das Betreuungspersonal lässt sowohl spezifische Formen der Krisenkonstruktion als auch besondere Formen kompetenter Krisenbewältigungsstrategien erkennen. Beide Aspekte zeigen, wie abschließend resümiert werden soll, Professionalisierungspotentiale an.

\section{Robotergestützte Demenzbetreuung als Forschungsfeld}

Angeleitet durch ein Grundinteresse an Technik in der Pflege haben wir 2013 eine ethnographische Studie in einem Altenpflegezentrum eines kirchlichen Trägers begonnen, das sich dadurch auszeichnet, dass hier in der Demenzbetreuung u. a. avancierte Technologie zum Einsatz kommt.

\subsection{Zur Technik}

Bei dieser Technologie handelt es sich um einen robbenförmigen, d. h. zoomorphen Roboter, den Kolling et al. (2013) als „sozial assistiv“ kategorisieren. Er ist seit 2005 in Japan und seit 2009 in Europa und den USA auf dem Markt und wird vornehmlich in der stationären Alten- und Demenzpflege eingesetzt. Lediglich im Herkunftsland Japan befinden sich über die Hälfte der verkauften Exemplare im Besitz von Privatkunden (Shibata 2012, S. 2530).

Nicht nur das Robotische, sondern die Technikhaftigkeit schlechthin entzieht sich bei diesem Gerät auf den ersten Blick. Denn das aus einer Reihe von Hard- und Softwarekomponenten bestehende ,technische (Sach)system“ (Ropohl 2009 [1979]; vgl. auch Weyer 2008) ist hinter einem weißen - dem Einsatzgebiet des Gesundheitssektors entsprechend antibakteriellen - Kunstfell verborgen. Das Gerät mutet deshalb zunächst wie ein (besonders körpernahes) Spielzeug, nämlich wie ein Kuscheltier an. Irritiert wird diese Anmutung allerdings, sobald man das Gerät hochnimmt, da 
es mit seinen fast $3 \mathrm{~kg}$ vom Gewicht her an einen Säugling erinnert, was durchaus beabsichtigt ist. Sobald das Gerät angeschaltet ist, verursachen die Aktuatoren, die den „Kopf“, die „Seitenflossen“ und die „Schwanzflosse“ bewegen, zudem leise Motorengeräusche.

Die Technik zeichnet sich dadurch aus, dass im Zusammenwirken der aus diversen Sensoren, Lautsprechern, Aktuatoren und Prozessoren bestehenden HardwareKomponenten mit einem auf Software basierenden Verhaltensmodell sogenannte „proactive and reactive processes“ initiiert werden können. Im Verstande einer solchen „Eigensteuerung“"3 simuliert das technische Artefakt zum einen Re-Aktionen auf Berührungen und plötzliche, laute Umgebungsgeräusche. Zum anderen ruft das Zusammenwirken der Software mit den Aktuatoren den Eindruck hervor, dass der Roboter von sich aus aktiv wird, d. h. seine Extremitäten und Augenlider ohne fremdes Zutun bewegt.

$\mathrm{Zu}$ den motorischen kommen auditive „Äußerungsformen“ hinzu, bei denen tierähnliche Geräusche simuliert werden, die sich als Wohl- oder Missfallenslaute interpretieren lassen. Das Gerät ist also so konstruiert, dass es sich (stoff-)tierähnlich anfühlt und anhört, und soll zugleich den Eindruck erwecken, zum Hören und Fühlen befähigt zu sein. Im Unterschied zu den in diesem Sinne nach zwei Seiten offenen taktilen und auditiven ist der visuelle „Kanal“ (vgl. von Scheve 2014) insofern reduziert, als der Roboter nicht mit einer Kamera, sondern lediglich mit Licht-Sensoren ausgestattet ist, womit zwar die Simulation eines Tag-Nacht-Rhythmus, aber kein Bildempfang (,Sehen“) möglich ist. Allerdings bieten die Aktuatoren in den Augenlidern dem Betrachter die Möglichkeit, das eigentätige Öffnen und Schließen der einem Babyface entsprechend überdimensioniert großen Augen als „Schauen“ bzw. „Angeschautwerden“ zu interpretieren.

Aufgrund seiner auf Berührungen und Ansprache ausgerichteten technischen Funktionen, die hinsichtlich der ,,appearance“ ${ }^{4}$ durch den Fellüberzug des tierförmigen Korpus und dessen Beweglichkeit unterstützt werden, ist das technische Artefakt in der sogenannten ,emotionalen Robotik“ (vgl. Meyer 2011; Klein et al. 2013) zu verorten, die in der Tradition des ,,affective computing“ (Picard 1997, 2003) steht: Denn das Design hebt zum einen auf die - insbesondere auditive - Darstellung eines ,emotionalen Zustands“ des Roboters ab und ist zum anderen darum bemüht, den Anschein einer Reaktion auf die - insbesondere taktil - zum Ausdruck gebrachten Emotionen des menschlichen Gegenübers zu erwecken. Im Design ist die Gestaltetheit des Roboters mit dessen gestaltender Wirkung verknüpft (vgl. Häussling 2010, S. 144). Mit der Gestalt(ung) geht eine Aufforderung zu einer bestimmten

\footnotetext{
3 Lindemann (2005, S. 131) spricht - in vorsichtiger Abgrenzung von „Selbststeuerung“ - von „Eigensteuerung“. Das scheinbare „Von-sich-aus-Aktivwerden“ des Artefakts ist jedoch eine (mitunter unbeabsichtigte) Nebenfolge menschlichen Handelns, so wie jede „Eigentätigkeit“ des Roboters genetisch auf menschliches Handeln (statt auf Handlungsträgerschaft von Technik) zurückzuführen ist, weil das technische Artefakt - bis hin zu jener Software, die dieses „lernen“ lässt - entsprechend programmiert worden ist.

${ }^{4}$ Einem postphänomenologischen Verständnis nach ist Technik nicht ontologisch, sondern von ihrer Erscheinung her zu bestimmen: „What matters for the human-robot relation is how the robot appears to human consciousness“ (Coeckelbergh 2011, S. 199; vgl. grundlegend Ihde 1990).
} 
Nutzungsweise einher, wobei diese Gebrauchsintention unterlaufen werden kann. ${ }^{5}$ Beides, die zweckentfremdete als auch die zweckdienliche Verwendung von Technik bringen „neue Sozialpraktiken im Alltag“ (Bonsiepe 1996, S. 25) hervor.

Mit dieser Perspektive auf sozio-technische Arrangements stellen wir das materielle Artefakt in den Mittelpunkt der Betrachtung. Allerdings wird es nicht zu einer handelnden Entität stilisiert, sondern - im wissenssoziologischen Verstande einer Objektivation (vgl. Pfadenhauer 2014b, 2014c) - als Teilelement menschlichen Handelns begriffen, das häufig zum Bezugspunkt wechselseitigen Wirk-Handelns avanciert.

\subsection{Zum Forschungsfeld}

Mit diesem wissenssoziologischen Grundverständnis erforschen wir die Verwendung der Roboter-Robbe in der stationären Demenzbetreuung. Im von uns untersuchten Pflegezentrum wird diese Technik ausschließlich von sogenannten ,zusätzlichen Betreuungskräften“ eingesetzt. Dabei handelt es sich um eine erst vor wenigen Jahren eingeführte berufliche Tätigkeit, die mit einem einwöchigen Orientierungspraktikum, 160 Ausbildungsstunden und einem zweiwöchigen Betriebspraktikum eine niedrig bemessene Qualifizierung voraussetzt.

Der Einsatz eines Roboters zur Betreuung und Aktivierung von Heimbewohnern ist im Katalog der in $\S 87 b$ Abs. 3 Sozialgesetzbuch XI aufgeführten Betreuungsund Aktivierungsmaßnahmen nicht explizit aufgeführt. Der Liste beispielhaft genannter Maßnahmen, mit denen Betreuungskräfte Heimbewohner mit besonderem Betreuungsbedarf zu „Alltagsaktivitäten motivieren sollen“, ist allerdings ein Passus vorangestellt, wonach grundsätzlich „Maßnahmen und Tätigkeiten in Betracht [kommen], die das Wohlbefinden, den physischen Zustand oder die psychische Stimmung der betreuten Menschen positiv beeinflussen können“ ( 2 Abs. 1 Betreuungskräfte$\mathrm{RI})$.

Im Zuge der von der Heimleitung getroffenen Entscheidung zum Erwerb des Roboters, die vom kirchlichen Heim-Träger mitgetragen wird, haben zwei der in der Pflegeeinrichtung angestellten ,zusätzlichen Betreuungskräfte“ eine vom Vertreiber des Geräts in Deutschland angebotene Einführung in das Gerät erhalten. ${ }^{6}$ Vor ihrem Ausscheiden hat eine der beiden Betreuungskräfte, die zwischenzeitlich den Arbeitgeber gewechselt hat, ihre aus der Anwendung des Geräts gewonnenen Erfahrungen $^{7}$ einer dritten Betreuerin vermittelt, womit das Gerät, von dem die Einrichtung zwei Exemplare besitzt, während unserer Feldforschung kontinuierlich von zwei ,zusätzlichen Betreuungskräften“ nach $§ 87$ b eingesetzt worden ist.

\footnotetext{
5 Vgl. zu dieser im Kontext der Cultural Studies als „,Aneignung“ und von uns im Hinblick auf ,Zweckentfremdung“ beobachteten Eigenwilligkeit Eisewicht und Pfadenhauer (2016).

6 Nicht nur in Deutschland forcieren die Vertriebsorganisationen eine Abgabe des technischen Geräts in Kombination mit einem Anwendertraining. Unsere Ethnographie bestätigt die These von Pedersen (2011, S. 44), dass hierbei neben dem richtigen Gebrauch auch die Einstellung zu dieser Technologie geschult wird.

7 Sie hatte auch ein vom Vertreiber initiiertes regionales Anwendertreffen besucht, das - ebenso wie die vom Vertreiber eingerichtete interaktive e-Lernplattform - dem Erfahrungsaustausch dienen sollte.
} 
Die teilnehmende Beobachtung und videographische Dokumentation dieser (Gruppen-) Aktivierung mit dem Roboter ist Kern unseres Datenmaterials. ${ }^{8}$ Den aus Videoaufnahmen, Fotos, ${ }^{9}$ Mitschnitten informeller Gespräche und Teambesprechungen sowie Beobachtungsprotokollen bestehenden Datenfundus haben wir um explorative Interviews mit Personen erweitert, die für die Aktivierung von Menschen mit Demenz einerseits und für den Vertrieb und die Verbreitung des Geräts andererseits zuständig sind. ${ }^{10}$ Dabei geht es uns nicht darum, personale Typen zu bilden, sondern um die (Ideal-)Typisierung situativer Vollzugsweisen. Hierfür betrachten wir die mit der Kamera aufgezeichneten Aktivierungseinheiten fallweise auf jene Eigenschaften hin, die in Bezug auf den Robotereinsatz charakteristisch sind und seine Dynamik ausmachen (vgl. Lueger 2000, S. 57 f.). Die nachfolgend kontrastierten Handlungsablauftypen beruhen auf der Verdichtung dieser Fälle $\mathrm{zu}$ idealisierten, von Abweichungen weitgehend bereinigten Grundtypen, deren wesentliche Eigenschaften dergestalt modellhaft hervortreten.

\section{Die Performanz der Betreuungskräfte}

Der Einsatz des Roboters hat in der von uns untersuchten Einrichtung unter den vielen Aktivierungsmaßnahmen, bei denen Klangschalen ebenso wie der Snoezelen-Wagen zum Einsatz kommen, einen festen Platz. Die Arbeit mit der künstlichen Robbe ist also neben diesen und Aktivitäten wie Erinnerungs-Frühstück, Gottesdienst, Theater und Aktivgruppen bis hin zur Aktivierung mit Hunden ein fester Programmpunkt. In den meisten Fällen handelt es dabei um eine sogenannte „Gruppenaktivierung“. ${ }^{11}$

Signifikant für die Handhabung dieses Roboters ist im Unterschied zur Automobilität vieler Prototypen, dass er sich nicht selbsttätig in einen Raum hineinbewegt. Er muss vielmehr dorthin gebracht werden, und empirisch finden wir hier unterschiedliche Tragetechniken. Das ist deshalb bemerkenswert, weil die Art, wie die Betreuerinnen das Gerät tragen, den Heimbewohnern in unterschiedlichem Maß Handlungsmöglichkeiten eröffnet: Alle von uns begleiteten qualifizierten Betreuungskräfte führen das Gerät nicht nebensächlich mit sich und sie transportieren es

\footnotetext{
8 Zur teilnehmenden Beobachtung vgl. Lueger (2010) und zur Videographie Tuma et al. (2013). Weniger intendiert als durch die Situation im Feld bedingt tendiert die teilnehmende Beobachtung immer wieder zur beobachtenden Teilnahme (vgl. hierzu Pfadenhauer und Grenz 2015; Hitzler 2007). Anstelle von Beobachtungsdaten gewinnen wir hierbei Erlebnisdaten, die insbesondere hinsichtlich der Erfahrung von Kommunikationsbrüchen und deren Überbrückung mittels Technik wertvoll sind. Deshalb bauen wir das Forschungsdesign derzeit systematisch in Richtung einer lebensweltanalytischen Ethnographie der Aktivierung aus.

9 Zum visuellen Protokollieren, innerhalb dessen dem Foto die Funktion einer Wissensform zukommt, (vgl. Pfadenhauer 2016).

10 Eine Kontextualisierung, deren Ergebnisse hier noch nicht berichtet werden können, erfährt unsere Forschung dadurch, dass wir uns dem Einsatz des Roboters in der ambulanten Altenpflege (Besucherdienste in der Schweiz) zuwenden.

11 Das entspricht den rechtlichen Vorgaben, wonach ,zur Prävention einer drohenden oder bereits eingetretenen sozialen Isolation Gruppenaktivitäten [...] das für die Betreuung und Aktivierung geeignete Instrument [sind]“" (Betreuungs-RI § 2 Abs. 3).
} 
schon gar nicht in dem für seine Aufbewahrung genutzten Karton durchs Haus. Sie tragen das Gerät vielmehr demonstrativ im Arm, was dazu führt, dass Bewohner von sich aus (auf) das Gerät ansprechen können. ${ }^{12}$ Dies bezeichnen wir als „Aktivierung im Vorübergehen“. Diese kommt insbesondere dann zustande, wenn die Betreuungskraft zusätzlich ihren Schritt verlangsamt und das Gerät bereits vor der Begegnung eingeschaltet hat. Im Hinblick darauf ist uns der sogenannte „Fliegergriff“ aufgefallen, bei dem der „Kopf“ der künstlichen Robbe in der Ellenbeuge des Tragarms platziert ist und die ,Schwanzflossen“, zwischen denen sich der An/Aus-Knopf des Geräts angebracht ist, gut in der Hand liegen: Gegenüber der häufiger beobachtbaren - Positionierung des Geräts wie ein Paket unter dem Arm hat diese in Bezug auf Säuglinge auch als Kolik-Griff bekannte Haltetechnik den Vorteil, dass der Schalter einfach erreichbar ist und das Gerät damit relativ unauffällig in Betrieb genommen werden kann. Dieser Griff wird im vom Geräte-Vertreiber aufgelegten ,Zertifizierungstraining“ zur „Bedienung“ empfohlen. Von den beiden Betreuungskräften, die dieses Training absolviert haben, wird der Griff von jener Person angewendet, d.h. als Wissen übernommen, bei der sich dieser schon beim Tragen ihrer Kinder bewährt hat.

Bei einer Bewohnerin oder einer Gruppe von Bewohnern angekommen, nimmt die Handlungsform des Tragens gestisch signifikant die Form des Darreichens (Anbietens) an. Dieser Übergang von der Demonstration zur Darreichung ist universell, d. h. unseren Beobachtungen zufolge unabhängig sowohl von der betreuenden als auch von der zu betreuenden Person. Nicht nur aufgrund dieser Verallgemeinerbarkeit über die einzelnen Betreuungspersonen und diversen Betreuungssituationen hinweg sprechen wir von Performanz. Offensichtlich wird der Roboter nicht schlicht eingesetzt, sondern die Betreuerinnen führen zusätzlich gewissermaßen vor, dass sie etwas (Besonderes) mit- bzw. zum Einsatz bringen.

Wenn die Betreuerin mit dem Gerät an Bewohner herantritt, die in einer Sitzgruppe oder an einem Tisch zusammensitzen - ja selbst, wenn mehrere Personen in dieser Gesellschaft reagieren - nimmt die Aktivierung auch in einer solchen Gruppe nicht die Gestalt eine Gruppenaktivierung an. D. h. im Unterschied zur Begrüßung adressiert die Betreuungskraft nun nicht mehr alle, sondern wendet sich mit dem Gerät im Arm einer Bewohnerin besonders (und je nach Reaktion unterschiedlich lange) zu. ${ }^{13}$ Solange die Betreuungskraft das Gerät auf diese Weise hin-hält, ist für die jeweilige Bewohnerin die situative Aufmerksamkeit sichergestellt - und zwar auch dann, wenn kein Gespräch zustande kommt. Mit diesem gestischen Aufrechterhalten der Situation, das die Betreuerin mimisch und verbal unterstützt, eröffnet sie gleichsam einen Raum für Kommunikation, den die Bewohnerin in Anspruch nehmen kann, aber nicht muss. Selbst in der Gruppenkonstellation nimmt der Robo-

\footnotetext{
12 Dabei darf nicht übersehen werden, dass nur aus der Perspektive eines ,normalen, hellwachen Erwachsenen“ (Kotsch und Hitzler 2013, S. 17; vgl. zur Abweichung infolge demenzieller Erkrankung Honer 2011) der mit einem weißen Kunstfell überzogene, robbenförmige Korpus aufgrund seiner Größe und Farbe praktisch unübersehbar ist. Bei einem altersbedingt eingeschränkten Sehvermögen, zu dem mit der Demenz auch noch eine Unempfänglichkeit für die ,Farbe“ Weiß hinzukommen kann, ist dies nicht zwangsläufig gegeben.

13 Wir können das Auswahlkriterium noch nicht identifizieren; es ist aber wahrscheinlich, dass hier aufgrund der Anwesenheit der Forscher ein ,Vorführmotiv“ die Auswahl zumindest mitbestimmt.
} 
tereinsatz also die Form einer Einzelaktivierung an. Diese kann sich unterschiedlich gestalten, wobei es zunächst vor allem einen Unterschied macht, ob der Betreuerin eine Kontaktaufnahme mit der Bewohnerin gelingt, d. h. ob sie auf ihre Darreichung des Geräts, die immer mit einer direkten Ansprache einhergeht, überhaupt eine Reaktion erzielt, die durch das Robotische, d.h. das Spezifische des Geräts bedingt sein kann, aber nicht muss. Im Fall einer erfolgreichen Kontaktaufnahme können wir zwei Darreichungstypen kontrastieren:

In Variante 1 setzt die Betreuerin immer wieder einen Gesprächsstimulus, greift einen abgerissenen Gesprächsfaden wieder auf, fordert zum Streicheln des Fells auf, kommentiert die Äußerungsformen des Roboters oder lädt zu deren Deutung ein. Dies ähnelt der Alltagssituation mit Haustieren, für die typisch ist, dass die direkte Ansprache des Tiers in der Regel rasch zum Gespräch über das Tier, von dort aus $\mathrm{zu}$ anderen Themen überwechselt - und wieder auf das Tier zurückspringt, wenn es sich bemerkenswert verhält oder das Gespräch stockt (vgl. Bergmann 1988).

Im Variante 2 hält sich die Betreuerin dagegen fast vollständig zurück. Ihre Haltung erinnert an die eines Psychoanalytikers, der durch die sich selber auferlegte Zurückhaltung eine künstliche Gesprächsatmosphäre erzeugt, die beim Patienten einen Erzählzwang evoziert. Sie agiert hier statt als Gesprächspartnerin als Beobachterin, deren Blick zwischen Bewohnerin und Gerät hin- und herwechselt. Dabei deutet sie in dieser für sie kommunikativ handlungsentlasteten Situation, wie die Bewohnerin den Roboter deutet bzw. ob die Bewohnerin einen Zusammenhang zwischen ihren Handlungen und der Selbsttätigkeit des Geräts herstellt. Dies gibt Raum für die Art von freischwebender Aufmerksamkeit, die Freud für die Psychoanalyse beschreibt, bei der es gerade nicht auf Fokussierung ankommt, sondern darauf, alle Details wichtig zu nehmen (vgl. Breidenstein et al. 2014, S. 89). So wie für die freudianische Psychoanalyse eine Sitzordnung typisch ist, in der der Therapeut neben oder hinter dem (Kopf des) Patienten platziert ist, ist die Positionierung der Betreuungskraft bemerkenswert: Für die Beobachterrolle begibt sie sich typischerweise in die Hocke schräg gegenüber der sitzenden Bewohnerin und hat damit sowohl den Blick auf den Roboter als auch und vor allem in das Gesicht der Bewohnerin.

Für die Rolle der Gesprächspartnerin ist hingegen keine besondere Anordnung auffällig. In der Ausübung dieser Rolle öffnet sich das Gespräch durchaus auch für weitere Bewohner. Diese werden keineswegs nur auf Betreiben der Betreuerin, sondern auch von der Bewohnerin in die technikvermittelte Zweierkonstellation hineingeholt. Diese Konstellation lässt sich als „,mediatisiert“ (Pfadenhauer und Dukat 2014) etikettieren, wenn man den Roboter als Medientechnologie versteht, mittels derer sich die ursprünglich unmittelbare Face-to-face-Konstellation und damit auch die Erfahrungen der beteiligten Personen verändern, worauf insbesondere die Ausdifferenzierung einer Beobachterrolle für die Betreuungspersonen ein Hinweis sein könnte.

\section{Professionalisierung durch Technik?}

Wir beobachten zwei Varianten des Technik-Einsatzes: Bei der einen Variante agiert die Aktivierungskraft als Teilnehmerin (des Gesprächs mit der Bewohnerin), bei 
der anderen als Beobachterin (des Umgangs der Bewohnerin mit dem Roboter), wobei im letzteren Fall typischerweise die Bewohnerin und nicht wie sonst in aller Regel die Betreuerin das Thema vorgibt. Als Anzeichen für eine Professionalisierung der Aktivierung gilt uns die Performanz der Betreuungskräfte. Diese geben den Bewohnern das technische Artefakt dezidiert nicht einfach als Spielzeug in die Hand. Wir haben bislang also keine Anhaltspunkte für die in der Technikfolgenabschätzung befürchtete Substitution menschlicher Arbeitskraft durch Robotik (vgl. Krings et al. 2014).

Die Betreuerinnen verwenden das Artefakt aber auch nicht als irgendein Aktivierungswerkzeug. Im ersten Handlungsablauftypus fungiert es als eine Art „Dritter im Bunde“. Bei dieser Art der Verwendung beziehen sich die Betreuungskräfte in einer Weise auf die Technik, die postphänomenologisch als ,alterity relation“ (Ihde 1990, S. 97) gedeutet werden könnte: Auch wenn die Betreuerinnen in gewisser Weise von dem Gerät fasziniert erscheinen, erscheint uns jedoch die Deutung überzogen, dass ihnen die Technik als „quasi-otherness“ (ebd., S. 100) gegenübertritt. Es ist vielmehr das Nicht-Vorhersagen-Können, wann der Roboter überhaupt etwas und was er dann „tut“, das die Gesprächssituation, in der sie sich mit den Heimbewohnern befinden und in der ein technisches Artefakt eine besondere Rolle spielt, für die Betreuungskräfte ,,besondert“. Es ist eine Objektivation, die - im Unterschied etwa zu einem (funktionstüchtigen) Wecker, der zu einem vorhersehbaren Zeitpunkt schrillt - aufgrund ihrer Programmierung zu unvorhersehbaren Zeitpunkten unterschiedliche Emanationen hervorbringt, auf die die Bewohner unvorhersehbar reagieren oder aber nicht, was gerade deshalb, weil es untypisch ist, die Gesprächssituation zu einer nicht-alltäglichen macht.

Das Problem, das es aus Sicht der Betreuerinnen zu bearbeiten gilt, ist keine Kommunikationskrise, wie dies z. B. für Beziehungskonflikte der Fall ist, die sich in Vorwürfen wie „Wir können einfach nicht miteinander reden“ oder „Du hörst mir einfach nicht zu!“ äußern. Das Problem, das sich für bzw. aus Sicht der Betreuungskräfte stellt, bewegt sich aber ebenfalls auf der Ebene der mittleren Transzendenz: Als krisenhaft konstruieren sie den fehlenden Bezug von Personen mit Demenz zu ihrer Mit-Welt, d. h. das abhandengekommene Vermögen, anderen innere Vorgänge anzuzeigen bzw. die Anzeigen anderer entsprechend zu deuten.

Hier eröffnet der Einsatz des technischen Artefakts einen optionalen Kommunikationszeitraum, den die Betreuerin performativ relativ lange aufrechterhält, nämlich a) körperlich, d.h. durch ihre Körperhaltung und -positionierung, b) gestisch, d. h. dadurch, dass sie das Gerät darbietet und beim Ablegen auf dem Tisch oder (selten) auf dem Schoß der Bewohnerin selbst berührt, c) mimisch, d. h. durch Blickkontakt, und d) mitunter auch verbal, d. h. durch die thematische Bezugnahme auf den Roboter. Der das Gerät kennzeichnende Kuschelfaktor fungiert als physische Kontaktbrücke und die für einen Roboter symptomatische „Selbsttätigkeit“"14 liefert mit ihrem Überraschungsmoment beiden Beteiligten beständig ein Thema zur möglichen Bezugnahme, wenn das Gespräch zu versiegen droht. Genau das führt gerade für Personen, die sich in ihrem demenziellen Prozess befinden, nicht selten zum Ende der Begegnung - ohne dass dies von ihnen gewünscht sein muss. Unserer bisheri-

14 Knoblauch (1991, S. 39) spricht in Bezug auf die Bewegung der Wünschelrute von „Selbsttätigkeit“. 
gen Einschätzung nach zeichnet sich hier aber eher eine empathische Haltung denn professionelle Kompetenz in der Bearbeitung dessen ab, was als Handlungskrise im engeren Sinn, nämlich als Interaktionskrise definiert wird. Und auch wenn der Entwickler derzeit die Anerkennung des Roboters als therapeutisches Mittel voranzutreiben versucht (vgl. Shibata 2010, 2012), geht die Professionalisierung der Berufsrolle u. E. nicht zwangsläufig mit dessen Einsatz als Heilmittel einher.

Professionssoziologisch bemerkenswert erscheint uns der Einsatz der Technik im zweiten Handlungsablauftypus. Hier sind Mensch und Technik auf eine Weise aufeinander bezogen, die man mit Ihde (1990, S. 80) als „hermeneutic relation“ bezeichnen könnte. Denn es macht den Anschein, dass die Technik, indem sie Anzeichen hervorbringt, die zu interpretieren sind, der Betreuerin hilft, etwas über die Welt der Bewohnerin zu erfahren, das selbst nicht sichtbar ist. So besteht die Besonderheit dieser Technik aus Sicht einer Betreuungskraft darin, dass sich (nur) mit ihr „Herzenstüren der Erinnerung“ (Interview Okt. 2013) öffnen (lassen). Damit meint sie mehr als einen Zugang zu besonders wertvollen biographischen Erlebnissen, die durch die Demenz verschüttet waren: Sie konnotiert damit das Durchscheinen der durch den demenziellen Prozess verschütteten Persönlichkeit, d. h. der ,,persönlichen Identität“ (Luckmann 1979), die den prinzipiell exzentrisch positionierten Menschen in einem Verhältnis zur Welt feststellt.

Die ,professionelle Leistung“ (Mieg und Pfadenhauer 2003) der Betreuerin in dieser Variante des Handlungsablaufs besteht in einer gezielten Selbst-Zurücknahme, die Bewohnerinnen erst zum Agieren (statt „nur“ Reagieren) in die Lage versetzt. Die Krise, die sich aus Sicht der Betreuerinnen hier konstituiert, ist nicht Demenz, was Aktivierungskräfte ebenso wie Pflegekräfte ohnehin als unzulängliche Diagnose erachten. Damit stellen sie die gesellschaftliche Konstruktion einer medizinisch definierten Wirklichkeit von Demenz keineswegs infrage. Ihre durch PraxisErfahrung ausgebildete Expertise lässt vielmehr die Ungenauigkeit bzw. mangelnde Spezifizierung der Diagnose „Demenz“ erkennen. Individualisierung ist nicht nur, aber insbesondere auch in Bezug auf Demenz ein wichtiger Aspekt, der Fachleute von ausgesprochen individuellen „Krankheits-“Verläufen bzw. Demenzprozessen sprechen lässt.

Diese Performanz weist insofern auf Kompetenz hin, als das Handeln mit der augenfälligen Zurückgenommenheit eine nicht-alltägliche „Qualität sozialen Handelns“ (Pfadenhauer 2010) aufweist. In dem Maße, in dem diese Qualität in der Form von Wissen als eine bestimmte Wirkung intendierende und richtige Handhabung des technischen Artefakts an andere weitergegeben wird, ist der Aspekt von Angemessenheit konnotiert (vgl. Knoblauch 2010). Die Kompetenz im Umgang mit dem Gerät könnte gerade darin bestehen, dass dessen robotische Eigentätigkeit - in vorsichtiger Analogie zum Röntgenapparat gedacht, der Bilder vom Körperinneren liefert, die Deutungskompetenz erfordern - verbale und non-verbale Äußerungsformen hervorruft, die als Anzeichen für Persönlichkeitsmerkmale gelesen werden können.

Aus der Verwendung der eigengesteuerten Technik als Beobachtungsinstrument ließe sich dergestalt ein Wissen destillieren, das im Hinblick auf die konstruierte Krise als „esoteric knowledge“ legitimiert werden könnte. Wenn Technik dieser Art zu einer Institution (vgl. Rammert 2007) im Tätigkeitsfeld der Demenzbetreuung 
und für ihre Bedienung eine besondere Qualifikation zur Voraussetzung gemacht würde, wären mögliche Weichen für ihre Professionalisierung gestellt.

\section{Resümee}

Unsere Empirie liefert längst kein abschließendes Bild der Professionalisierung durch Technik, sondern ist, neben vielem anderen, durch eine Perspektive auf organisatorische Settings und Prozesse des Organisierens (vgl. Pfadenhauer 2008) zu ergänzen. ${ }^{15}$ Sowohl im Hinblick auf die Dienstleistungserbringung als auch auf die uns interessierende Institutionalisierung von Technik ist eine integrierende Perspektive auf Profession und Organisation unerlässlich (vgl. grundlegend bereits Klatetzki und Tacke 2005).

Sollte unsere These plausibel sein, dass Technik ein Professionalisierungselement bilden kann (ohne hiermit tatsächlich die Professionalisierung der Aktivierung bzw. Demenzbetreuung prognostizieren zu wollen), schließt sich die Frage an, ob sich eine Professionalisierung der Betreuungsrolle zwangsläufig gegen anrainende Berufsgruppen vollzieht. Als Konkurrentin im Kampf um Zuständigkeit liegt die Pflege nahe, aus deren Reihen unter Zugrundelegung eines ganzheitlichen Pflegeverständnisses Ansprüche angemeldet werden könnten (vgl. hierzu Dammert et al. 2016). Demgegenüber kommt der Vorstand des GKV-Spitzenverbands im Vorwort der von ihm in Auftrag gegebenen Evaluation der Tätigkeit der zusätzlichen Betreuungskräfte zu dem Befund,

dass die Zusammenarbeit zwischen Betreuungs- und „,normalen“ Pflegekräften nicht als Konkurrenz, sondern von beiden Seiten als bereichernd empfunden wird. Nahezu alle befragten Wohnbereichsleitungen sehen in den Betreuungskräften eine große Unterstützung und Entlastung im Alltag, der Einsatz der Betreuungskräfte verschaffe dem Personal mehr Zeit für die Pflege. Ebenso viele geben an, dass sich durch die zusätzlichen Helfer die Versorgung der Pflegebedürftigen insgesamt verbessert habe. (GKV-Spitzenverband 2012, S. 3)

Zweifel an diesem Befund lassen sich dahingehend anmelden, dass kaum Pflegekräfte, sondern lediglich Pflegedienst- und Wohnbereichsleitungen befragt wurden. Unsere ethnographischen Erkundungen fördern durchaus ein Spannungsverhältnis zwischen Pflege und Betreuung zutage, das sich sowohl in der alltäglichen Praxis als auch in der Qualifizierung der angehenden Betreuungskräfte manifestiert, die von ihren Ausbildern explizit zu einem ,,boundary management“", d. h. zu einer Grenzziehung hinsichtlich ihres Einsatzbereichs aufgefordert werden.

Eine Professionalisierungsperspektive darf nicht außer Acht lassen, dass die Einführung der sogenannten ,qualifizierten Betreuungskraft“ in $§ 87 b$ des Sozialgesetz-

\footnotetext{
15 ,What is evidentially missing are articles dealing with the meso-level, that is the consequences of an integration of robots in organizational settings. The introduction of a care-giving robot (e.g. Paro) will evidently not only create new human-robot-interactions, but will also change the organizational setting in nursing homes with respect to workload, work description and hierarchies“ (Meister 2014, S. 113).
} 
buchs VI auch und nicht zuletzt auf Personalmissstände im Pflegebereich und als Arbeitsbeschaffungsmaßnahme auf die Lage am deutschen Arbeitsmarkt reagiert. Zum anderen darf der weite Weg hin zu einer Profession nicht unterschätzt werden, den Wilensky (1972, S. 204) im Anschluss an Hughes (1964) als „Naturgeschichte der Professionalisierung“ beschrieben hat: An deren Anfang steht der Übergang von einer Nebentätigkeit zur Vollberuflichkeit mit eigenem Arbeitsbereich, der an eine Ausbildung geknüpft wird, für die eigene Schulen eingerichtet werden, die einen akademischen Status zu erreichen versuchen. Am Ende dieses Prozesses, der nur bei wenigen Berufen zum Abschluss kommt, stehen die Etablierung von Berufsverbänden, die staatliche Lizenzierung des Berufsmonopols und die Formulierung einer Professionsethik (vgl. ebd., S. 205). Es scheint übertrieben, für die Demenzbetreuung mit ihren minimalen Qualifizierungsmaßnahmen diesbezüglich auch nur ein Anfangsstadium zu veranschlagen. Sehr wahrscheinlich ist, dass sie den Verlauf nehmen wird, den Marshall (1939) für den „Semi-Professionalismus“ prognostiziert hat, wonach ,das Qualifikationsniveau steigen und die Verbindlichkeit von Kriterien verantwortungsvoller und kompetenter Berufsausübung zunehmen wird“ (Wilensky 1972, S. 212). Allerdings ist die Demenzbetreuung eng mit einer Institution, dem Seniorenpflegezentrum, verknüpft und setzt sich hier (zaghaft) gegen die Vereinnahmung durch anrainende Berufsgruppen zur Wehr, was Hughes (1964, S. 133) zufolge im Fall anderer Berufsgruppen (Sozialarbeiter, Krankenschwestern, Bibliothekare) für eine Professionalisierung ausschlaggebend war.

Aus dem Blickwinkel einer Professionalisierungslogik ist zudem bemerkenswert, dass die Definition von Demenz als Krankheit im Tätigkeitsbereich der Demenzbetreuung umstritten ist. Denn das Bemühen, dem medizinischen (und landläufigen) Verständnis eine eigene Bestimmung entgegenzusetzen, kann als Anzeichen einer eigenständigen Problembestimmung gedeutet werden. Statt von einer Demenzerkrankung sprechen Demenzbetreuer von „Menschen in ihrem jeweiligen demenziellen Prozess“", womit einerseits an die seit Längerem in die Medizin vorgedrungene Individualisierung angeschlossen wird, andererseits aber in Kritik an einer Pathologisierung des Alterns Demenz als „natürlicher“ Bestandteil des Alterungsprozesses akzentuiert wird.

Gerade für die Startphase von Professionalisierung betont Hughes (1964, S. 137) die Bedeutung der von ihm sogenannten ,enthusiastic mavericks“. Solche Personen, die vehement eine andere als die gängige Sicht vertreten, finden sich eben auch in der Demenzbetreuung. Sie legen keinerlei Berührungsängste mit Technik im Allgemeinen und der sozialen Robotik im Besonderen an den Tag, wobei sie Letzterer explizit die Qualität eine „Türöffners“ zusprechen. Dies wird insbesondere in jener Variante deutlich, bei der sich die Betreuungskräfte auf eine Beobachterrolle einlassen und die Eigentätigkeit der robotischen Technik nutzen, um die Heimbewohner zum Agieren anzuregen. Die Betreuungskräfte fungieren hier einerseits als Krisenkonstrukteure, indem sie die ungenaue medizinische Diagnose „Demenz“ gemäß ihrer eigenen Handlungslogik situativ spezifizieren. Andererseits steht ihnen mit der ,assistiven“ Verwendung der Robotertechnik eine Krisenbewältigungsstrategie zur Verfügung, die sich im Hinblick auf das von ihnen etablierte Krisenverständnis als angemessen erweist. Die Kombination dieses Personentypus mit einem diese Technik instrumentalisierenden Handlungsablauftypus birgt Professionalisierungspotential. 
Open access funding provided by University of Vienna.

Open Access. Dieser Artikel wird unter der Creative Commons Namensnennung 4.0 International Lizenz (http://creativecommons.org/licenses/by/4.0/deed.de) veröffentlicht, welche die Nutzung, Vervielfältigung, Bearbeitung, Verbreitung und Wiedergabe in jeglichem Medium und Format erlaubt, sofern Sie den/die ursprünglichen Autor(en) und die Quelle ordnungsgemäß nennen, einen Link zur Creative Commons Lizenz beifügen und angeben, ob Änderungen vorgenommen wurden.

\section{Literatur}

Berger, Peter L., und Thomas Luckmann. 1969. Die gesellschaftliche Konstruktion der Wirklichkeit. Eine Theorie der Wissenssoziologie. Übers. Monika Plessner. Frankfurt: Fischer.

Bergmann, Jörg R. 1988. Haustiere als kommunikative Ressourcen. In Kultur und Alltag, Hrsg. HansGeorg Soeffner, 299-312. Göttingen: Schwartz.

Bonsiepe, Gui. 1996. Interface. Design neu begreifen. Mannheim: Bollmann.

Breidenstein, Georg, Stefan Hirschauer, Herbert Kalthoff, und Boris Nieswand. 2014. Ethnografie. Die Praxis der Feldforschung. Konstanz: UVK.

Coeckelbergh, Marc. 2011. Humans, animals, and robots. A phenomenological approach to human-robot relations. International Journal of Social Robotics 3:197-204.

Dammert, Matthias, Christine Keller, Thomas Beer, und Helma Bleses. 2016. Person-Sein zwischen Anspruch und Wirklichkeit. Eine Untersuchung zur Anwendung der Integrativen Validation und der Basalen Stimulation in der Begleitung von Personen mit Demenz. Weinheim: Beltz Juventa.

Eisewicht, Paul, und Michaela Pfadenhauer. 2016. Zweckentfremdung als Movens von Aneignungskulturen. Circuit Bending oder: Der gemeinschaftsstiftende inkompetente Gebrauch von Spielzeug. In Zweckentfremdung. „Unsachgemäßer“ Gebrauch als kulturelle Praxis, Hrsg. Maria Dillschnitter, und David Keller, 155-174. München: Fink.

Endreß, Martin. 2014. Grußwort. Eröffnungsveranstaltung, 37. Kongress der Deutschen Gesellschaft für Soziologie, Trier, 6. Oktober 2014.

GKV-Spitzenverband (Hrsg.). 2012. Betreuungskräfte in Pflegeeinrichtungen. Berlin: GKV-Spitzenverband.

Häußling, Roger. 2010. Zum Design(begriff) der Netzwerkgesellschaft. Design als zentrales Element der Identitätsformation in Netzwerken. In Relationale Soziologie. Zur kulturellen Wende der Netzwerkforschung, Hrsg. Jan Fuhse, und Sophie Mützel, 137-162. Wiesbaden: VS.

Hitzler, Ronald. 2007. Ethnographie. In Qualitative Marktforschung, Hrsg. Renate Buber, und Hartmut Holzmüller, 207-218. Wiesbaden: Gabler.

Hitzler, Ronald. 2014. Ceteris non paribus. Subjektiv bedeutsame Lebensereignisse als Generatoren von Wissenskrisen. Vortrag. Sektion Wissenssoziologie, 37. Kongress der Deutschen Gesellschaft für Soziologie, Trier, 8. Oktober 2014.

Honer, Anne. 2011. Zeit-Konfusionen. Zur intersubjektiven Rekonstruktion des temporalen Erlebens Demenzkranker. In Kleine Leiblichkeiten. Erkundungen in Lebenswelten, S. 131-140. Wiesbaden: VS.

Hughes, C. Everett. 1964. Men and their work, 2. Aufl., Glencoe: Free Press.

Ihde, Don. 1990. Technology and the lifeworld: From garden to earth. Bloomington: Indiana University Press.

Klatetzki, Thomas, und Veronika Tacke (Hrsg.). 2005. Organisation und Profession. Wiesbaden: VS.

Klein, Barbara, Lone Gaedt, und Glenda Cook. 2013. Emotional robots: Principles and experiences with Paro in Denmark, Germany, and the UK. GeroPsych 26:89-99.

Knoblauch, Hubert. 1991. Die Welt der Wünschelrutengänger und Pendler. Erkundungen einer verborgenen Wirklichkeit. Frankfurt: Campus.

Knoblauch, Hubert. 2010. Von der Kompetenz zur Performanz. Wissenssoziologische Aspekte von Kompetenz. In Soziologie der Kompetenz, Hrsg. Thomas Kurtz, und Michaela Pfadenhauer, 237-255. Wiesbaden: VS.

Knoblauch, Hubert. 2014. Wissenssoziologie. 3. Aufl. Konstanz: UVK.

Kolling, Thorsten, Julia Haberstroh, Roman Kaspar, Johannes Pantel, Frank Oswald, und Monika Knopf. 2013. Evidence and deployment-based research into care for the elderly using emotional robots. $G e$ roPsych 26:83-88.

Koselleck, Reinhart. 1976. Krise. In Historisches Wörterbuch der Philosophie, Hrsg. Joachim Ritter, 1235-1240. Darmstadt: WBG.

Kotsch, Lakshmi, und Ronald Hitzler. 2013. Selbstbestimmung trotz Demenz. Ein Gebot und seine praktische Relevanz im Pflegealltag. Weinheim: Beltz Juventa. 
Krings, Bettina-Johanna, Knud Böhle, Michael Decker, Linda Nierling, und Christoph Schneider. 2014. Serviceroboter in Pflegearrangements. In Zukünftige Themen der Innovations- und Technikanalyse: Lessons learned und ausgewählte Ergebnisse, Hrsg. Michael Decker, Torsten Fleischer, Jens Schipps, und Nora Weinberger, 63-121. Karlsruhe: KIT Scientific Publishing.

Lindemann, Gesa. 2005. Die Verkörperung des Sozialen. Theoriekonstruktion und empirische Forschungsperspektiven. In Soziologie des Körpers, Hrsg. Markus Schroer, 114-138. Frankfurt: Suhrkamp.

Luckmann, Thomas. 1979. Persönliche Identität, soziale Rolle und Rollendistanz. In Identität, Hrsg. Odo Marquard, und Karlheinz Stierle, 109-120. München: Fink.

Luckmann, Thomas, und Walter Michael Sprondel. 1972. Einleitung. In Berufssoziologie, Hrsg. Thomas Luckmann, und Walter Michael Sprondel, 11-21. Köln: Kiepenheuer \& Witsch.

Lueger, Manfred. 2000. Grundlagen qualitativer Feldforschung. Wien: WUV.

Lueger, Manfred. 2010. Interpretative Sozialforschung. Die Methoden. Wien: WUV.

Luhmann, Niklas, und Karl-Eberhard Schorr. 1979. Reflexionsprobleme im Erziehungssystem. Stuttgart: Klett-Cotta.

Maiwald, Kai-Olaf. 2004. Professionalisierung im modernen Berufssystem. Das Beispiel der Familienmediation. Wiesbaden: VS.

Marshall, Thomas H. 1939. The recent history of professionalism in relation to social structure and social policy. Canadian Journal of Economics and Political Science 5:325-334.

Meister, Martin. 2014. When is a robot really social. An outline of the robot sociologicus. Science, Technology \& Innovation Studies 10:107-134.

Meyer, Sibylle. 2011. Mein Freund der Roboter. Berlin: VDE.

Mieg, Harald, und Michaela Pfadenhauer (Hrsg.). 2003. Professionelle Leistung - Professional Performance. Positionen der Professionssoziologie. Konstanz: UVK.

Nassehi, Armin. 2012. Der Ausnahmezustand als Normalfall. Modernität als Krise. In Krisen lieben: Kursbuch 170, 34-49. Hamburg: Murmann.

Oevermann, Ulrich. 1996. Theoretische Skizze einer revidierten Theorie professionalisierten Handelns. In Pädagogische Professionalität, Hrsg. Arno Combe, und Werner Helsper, 70-182. Frankfurt: Suhrkamp.

Oevermann, Ulrich. 2006. Wissen, Glauben, Überzeugung. Ein Vorschlag zu einer Theorie des Wissens aus krisentheoretischer Perspektive. In Neue Perspektiven der Wissenssoziologie, Hrsg. Dirk Tänzler, Hubert Knoblauch, und Hans-Georg Soeffner, 79-118. Konstanz: UVK.

Pedersen, Per Lyder. 2011. Do elders dream of electric seals? A SCOT analysis of the mental commitment robot PARO in elderly care. Masterarbeit. Universität Oslo.

Pfadenhauer, Michaela. 2003. Professionalität. Eine wissenssoziologische Rekonstruktion institutionalisierter Kompetenzdarstellungskompetenz. Opladen: Leske + Budrich.

Pfadenhauer, Michaela. 2005. Die Definition des Problems aus der Verwaltung der Lösung. Professionelles Handeln revisited. In Professionelles Handeln, Hrsg. Michaela Pfadenhauer, 9-26. Wiesbaden: VS.

Pfadenhauer, Michaela. 2008. Organisieren. Eine Fallstudie zum Erhandeln von Events. Wiesbaden: VS.

Pfadenhauer, Michaela. 2010. Kompetenz als Qualität sozialen Handelns. In Soziologie der Kompetenz, Hrsg. Thomas Kurtz, und Michaela Pfadenhauer, 149-172. Wiesbaden: VS.

Pfadenhauer, Michaela. 2014a. Der Kompetenzstreit um „Kompetenz“. Ein umkämpftes Konstrukt in wissens- und professionssoziologischer Perspektive. In Kompetenz, Performanz und soziale Teilhabe. Sozialpädagogische Perspektiven auf ein bildungstheoretisches Konstrukt, Hrsg. Stefan Faas, Petra Bauer, und Rainer Treptow, 41-50. Wiesbaden: VS.

Pfadenhauer, Michaela. 2014b. On the sociality of social robots. A sociology of knowledge perspective. Science, Technology \& Innovation Studies 10:137-163.

Pfadenhauer, Michaela. 2014c. Von Objekten zu Objektivierung. Technische Artefakte im Kommunikativen Konstruktivismus. Vortrag. Tagung „Under Construction. Perspektiven des kommunikativen Konstruktivismus“, Technische Universität Berlin, 14. November 2014.

Pfadenhauer, Michaela. 2015. The contemporary appeal of artificial companions. Social robots as vehicles to cultural worlds of experience. The Information Society 31:284-293.

Pfadenhauer, Michaela. 2016. Fotografieren (lassen) in der lebensweltanalytischen Ethnographie. Das Foto als Wissensform. In Fotografie und Gesellschaft. Phänomenologische und wissenssoziologische Perspektiven, Hrsg. Thomas Eberle. Bielefeld: transcript.

Pfadenhauer, Michaela, und Christoph Dukat. 2014. Künstlich begleitet. Der Roboter als neuer bester Freund des Menschen? In Unter Mediatisierungsdruck, Hrsg. Tilo Grenz, und Gerd Möll, 189-210. Wiesbaden: Springer VS.

Pfadenhauer, Michaela, und Tilo Grenz. 2015. Uncovering the essence: The why and how of supplementing observation with participation in phenomenology-based ethnography. Journal of Contempora- 
ry Ethnography. http://jce.sagepub.com/content/early/2015/05/28/0891241615587382.full.pdf+html. Zugegriffen: 05.06.2015.

Pfadenhauer, Michaela, und Alexa Kunz. 2010. Professionen. In Handbuch Wissensgesellschaft. Theorien, Themen und Probleme, Hrsg. Anina Engelhardt, und Laura Kajetzke, 235-246. Bielefeld: transcript.

Picard, Rosalind W. 1997. Affective computing. Cambridge, MA: MIT Press.

Picard, Rosalind W. 2003. Affective computing: Challenges. International Journal of Human-Computer Studies 59:55-64.

Prisching, Manfred. 1983. „Krise“ als gesellschaftlicher Prozeß und als geschichtsphilosophisches Problem. In Der Mensch und die Wissenschaften vom Menschen, Hrsg. Gerhard Frey, und Josef Zelger, 809-824. Innsbruck: Solaris.

Prisching, Manfred. 1986. Krisen. Eine soziologische Untersuchung. Wien: Böhlau.

Rammert, Werner. 2007. Die technische Konstruktion als Teil der gesellschaftlichen Konstruktion der Wirklichkeit. In Zur Kritik der Wissensgesellschaft, Hrsg. Dirk Tänzler, Hubert Knoblauch, und HansGeorg Soeffner, 83-100. Konstanz: UVK.

Ropohl, Günter. 2009 [1979]. Allgemeine Technologie: Eine Systemtheorie der Technik. Karlsruhe: Universitätsverlag.

Scheve, Christian von. 2014. Interaction rituals with artificial companions. From media equation to emotional relationships. Science, Technology \& Innovation Studies 10:65-83.

Schütz, Alfred. 2004 [1932]. Der sinnhafte Aufbau der sozialen Welt. Alfred Schütz Werkausgabe, Bd. 1. Konstanz: UVK.

Schütz, Alfred, und Thomas Luckmann. 2003 [1975]. Strukturen der Lebenswelt. Konstanz: UVK.

Shibata, Takanori. 2010. Integration of therapeutic robot, Paro, into welfare systems. In Proceedings of the 28th Annual European Conference on Cognitive Ergonomics (ECCE 2010), New York: Association for Computing Machinery.

Shibata, Takanori. 2012. Therapeutic seal robot as biofeedback medical device: Qualitative and quantitative evaluations of robot therapy in dementia care. IEEE Proceedings 100:2527-2538.

Tuma, René, Bernt Schnettler, und Hubert Knoblauch. 2013. Videographie: Einführung in die interpretative Videoanalyse sozialer Situationen. Wiesbaden: Springer VS.

Weyer, Johannes. 2008. Techniksoziologie: Genese, Gestaltung und Steuerung sozio-technischer Systeme. Weinheim: Juventa.

Whitehouse, Peter J., und Daniel George. 2009. Mythos Alzheimer: Was Sie schon immer über Alzheimer wissen wollten, Ihnen aber nicht gesagt wurde, Übers. Gabriele Kreutzner. Bern: Huber.

Wilensky, Harold L. 1972. Jeder Beruf eine Profession? In Berufssoziologie, Hrsg. Thomas Luckmann, und Walter Michael Sprondel, 198-215. Köln: Kiepenheuer \& Witsch.

Michaela Pfadenhauer Prof. Dr., ist seit 2014 Professorin für Kultur und Wissen am Institut für Soziologie der Universität Wien. Forschungsschwerpunkte: Phänomenologisch orientierte Wissenssoziologie, Sozialkonstruktivismus als Paradigma der Sozialwissenschaften, Kulturwandel und Mediatisierung, Herausforderungen der Social Robotics für Sozialität, Lebensweltanalytische Ethnographie. Ausgewählte Veröffentlichungen: Peter L. Berger. Reihe „Klassiker der Wissenssoziologie“. Konstanz: UVK 2010; Social Robots: Form, Content, Critique, Hrsg. zusammen mit Satomi Sugyama und Charles Ess. Theme Issue of The International Journal of Social Robotics; Gemeinwohlorientierung als Maxime professionellen Handelns. In: Handbuch Professionsentwicklung, Hrsg. Michael Dick, Winfried Marotzki und Harald Mieg, 40-50. Bad Heilbrunn: Julius Klinkhardt 2016.

Christoph Dukat M.A., ist wissenschaftlicher Mitarbeiter am Institut für Soziologie des Karlsruher Instituts für Technologie (KIT). Forschungsschwerpunkte: Wissenssoziologie, Social Robotics, Ethnographische Forschungsansätze, Abgrenzungen von Tätigkeitsfeldern in der Pflege und Betreuung dementer Menschen. Ausgewählte Veröffentlichungen: Robot Caregiver or Robot-Supported Caregiving? The Performative Deployment of the Social Robot PARO in Dementia Care. International Journal of Social Robotics 2015, 7:393-406 (gem. mit Michaela Pfadenhauer); Zur Wirkung von Technik - Ethnographisch gestützte Überlegungen am Beispiel der Social Robotics in der Demenzbetreuung In: Materiale Analysen - Methodenfragen in Projekten, Hrsg. Nicole Burzan, Ronald Hitzler und Heiko Kirschner, 33-52. Wiesbaden: Springer VS 2016 (gem. mit Michaela Pfadenhauer); Lebensweltliche und Fokussierte Ethnographie - Gegenseitige Befruchtung oder Begrenzung? In: Old School - New School? Beiträge der 5. Fuldaer Feldarbeitstage, Hrsg. Ronald Hitzler, Simone Kreher, Angelika Poferl und Norbert Schröer. Essen: Oldib 2016 (im Erscheinen). 